\title{
A Class II Aldolase Mimic
}

Jimmy Hedin-Dahlström, Jenny P. Rosengren-Holmberg, Sacha Legrand, Susanne Wikman and

\author{
Ian A. Nicholls*
}

Bioorganic and Biophysical Chemistry Laboratory, Department of Chemistry and Biomedical Sciences, University of Kalmar, SE-391 82 Kalmar, Sweden

\section{Correspondence to:}

Ian A. Nicholls

Email: ian.nicholls@hik.se

Tel: +46-480 446258

Fax: +46-480 446244

\section{Table of Contents}

Spectroscopic data for:

(1S, 4R)-Product (3a)

Page S2-S6

$(1 R, 4 S)$-Product (3b)

Page S7-S9

$(1 S, 3 S, 4 S)$-TSA (4a)

Page S10-S12

$(1 R, 3 R, 4 R)$-TSA (4b)

Page S13

Keto-enol tautomerism

Page S14

VIS-titrations

Page S15

Binding studies

Page S16

Swelling studies 
Supporting information:

Hedin-Dahlström, J.; Rosengren-Holmberg, J.P.; Legrand, S.; Wikman, S.; Nicholls, I.A. A Class II Aldolase Mimic

\section{(1S, 4R)-(E)-3-benzylidene-1,7,7-trimethylbicyclo[2.2.1]heptan-2-one (3a)}

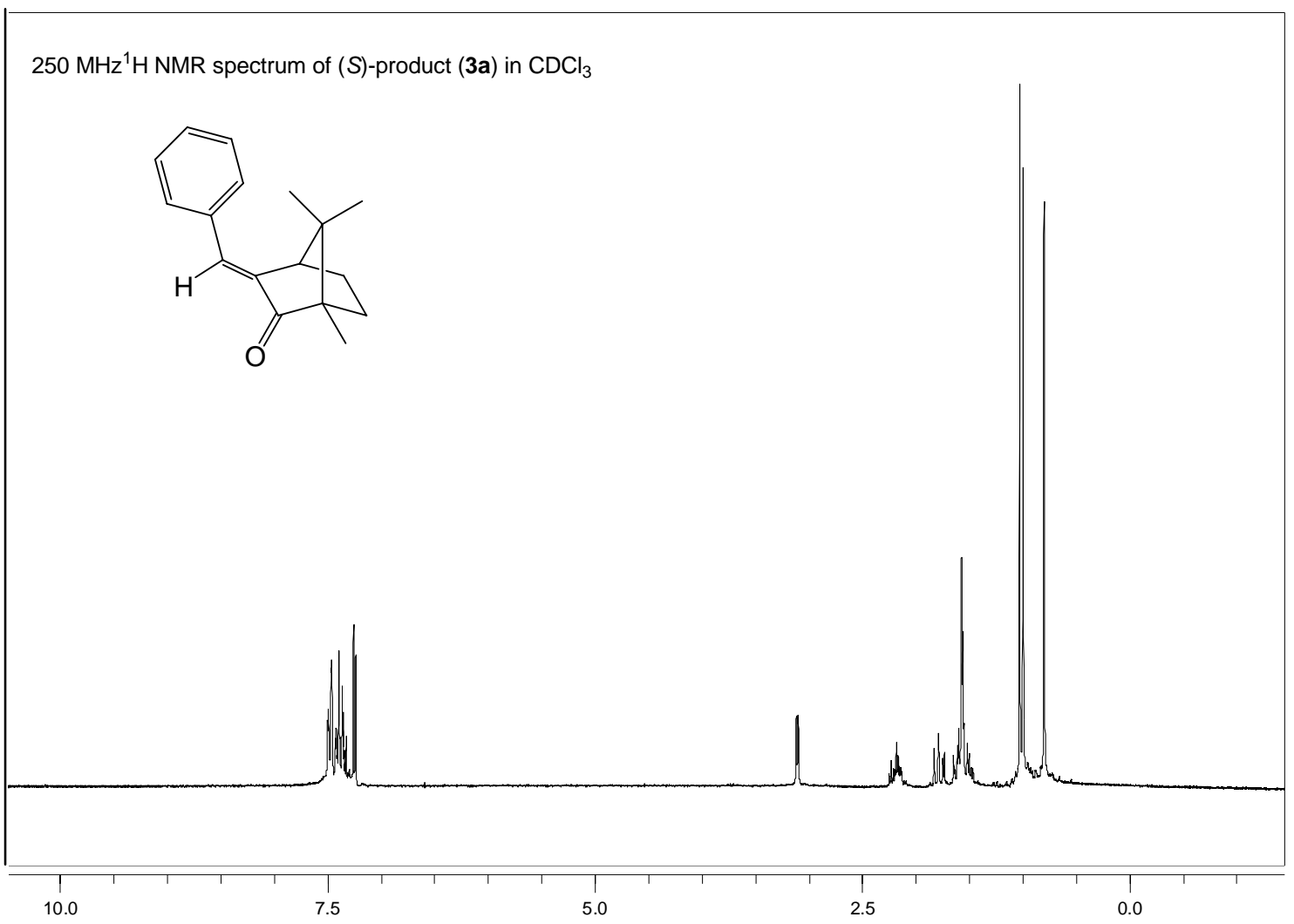

$\mathrm{ppm}$

$63 \mathrm{MHz}{ }^{13} \mathrm{C}$ NMR spectrum of (S)-product (3a) in $\mathrm{CDCl}_{3}$<smiles>CC12CCC(C(=Cc3ccccc3)C1=O)C2(C)C</smiles>

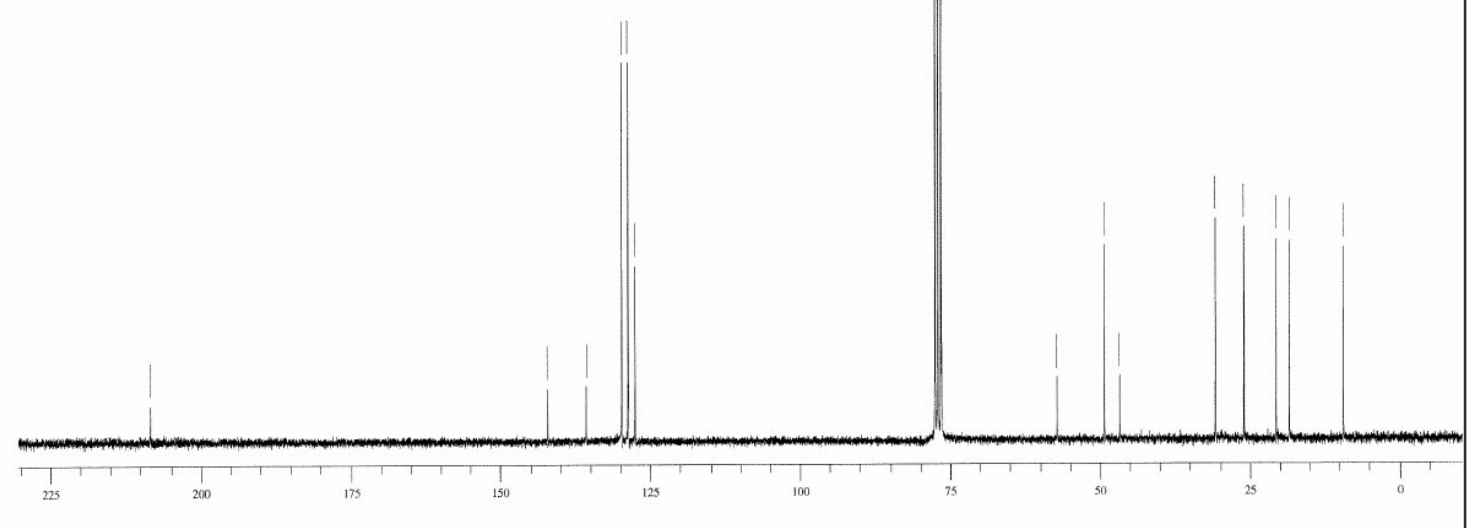


Supporting information:

Hedin-Dahlström, J.; Rosengren-Holmberg, J.P.; Legrand, S.; Wikman, S.; Nicholls, I.A. A Class II Aldolase Mimic

(1S, 4R)-(E)-3-benzylidene-1,7,7-trimethylbicyclo[2.2.1]heptan-2-one (3a)

$63 \mathrm{MHz}$ Dept-45 spectrum of (S)-product (3a) in $\mathrm{CDCl}_{3}$<smiles>CC12CCC(C(=Cc3ccccc3)C1=O)C2(C)C</smiles>

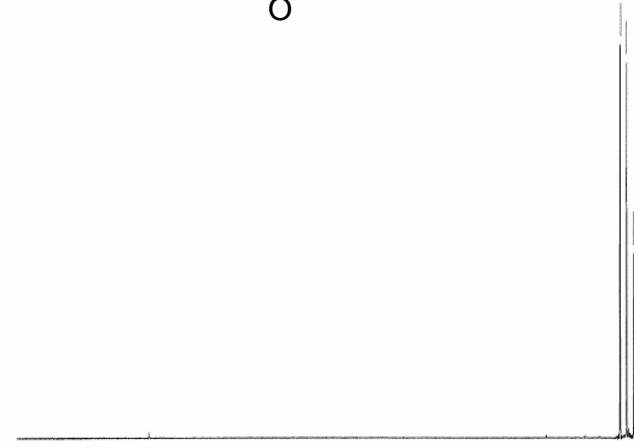

$\prod_{225}^{1}$

200

175

150

125

100

75

$63 \mathrm{MHz}$ Dept-90 spectrum of (S)-product (3a) in $\mathrm{CDCl}_{3}$

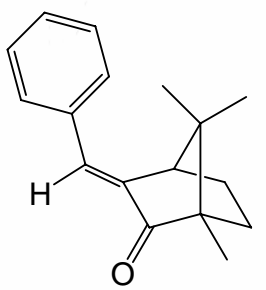


Supporting information:

Hedin-Dahlström, J.; Rosengren-Holmberg, J.P.; Legrand, S.; Wikman, S.; Nicholls, I.A. A Class II Aldolase Mimic

\section{(1S, 4R)-(E)-3-benzylidene-1,7,7-trimethylbicyclo[2.2.1]heptan-2-one (3a)}

$63 \mathrm{MHz}$ Dept-135 spectrum of (S)-product (3a) in $\mathrm{CDCl}_{3}$

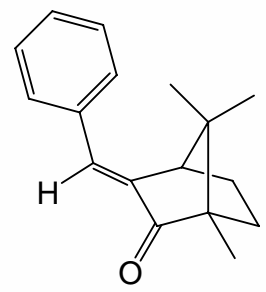


Supporting information:

Hedin-Dahlström, J.; Rosengren-Holmberg, J.P.; Legrand, S.; Wikman, S.; Nicholls, I.A. A Class II Aldolase Mimic

(1S, 4R)-(E)-3-benzylidene-1,7,7-trimethylbicyclo[2.2.1]heptan-2-one (3a)

$400 \mathrm{MHz}$ COSY spectrum of (S)-product (3a) in $\mathrm{CDCl}_{3}$

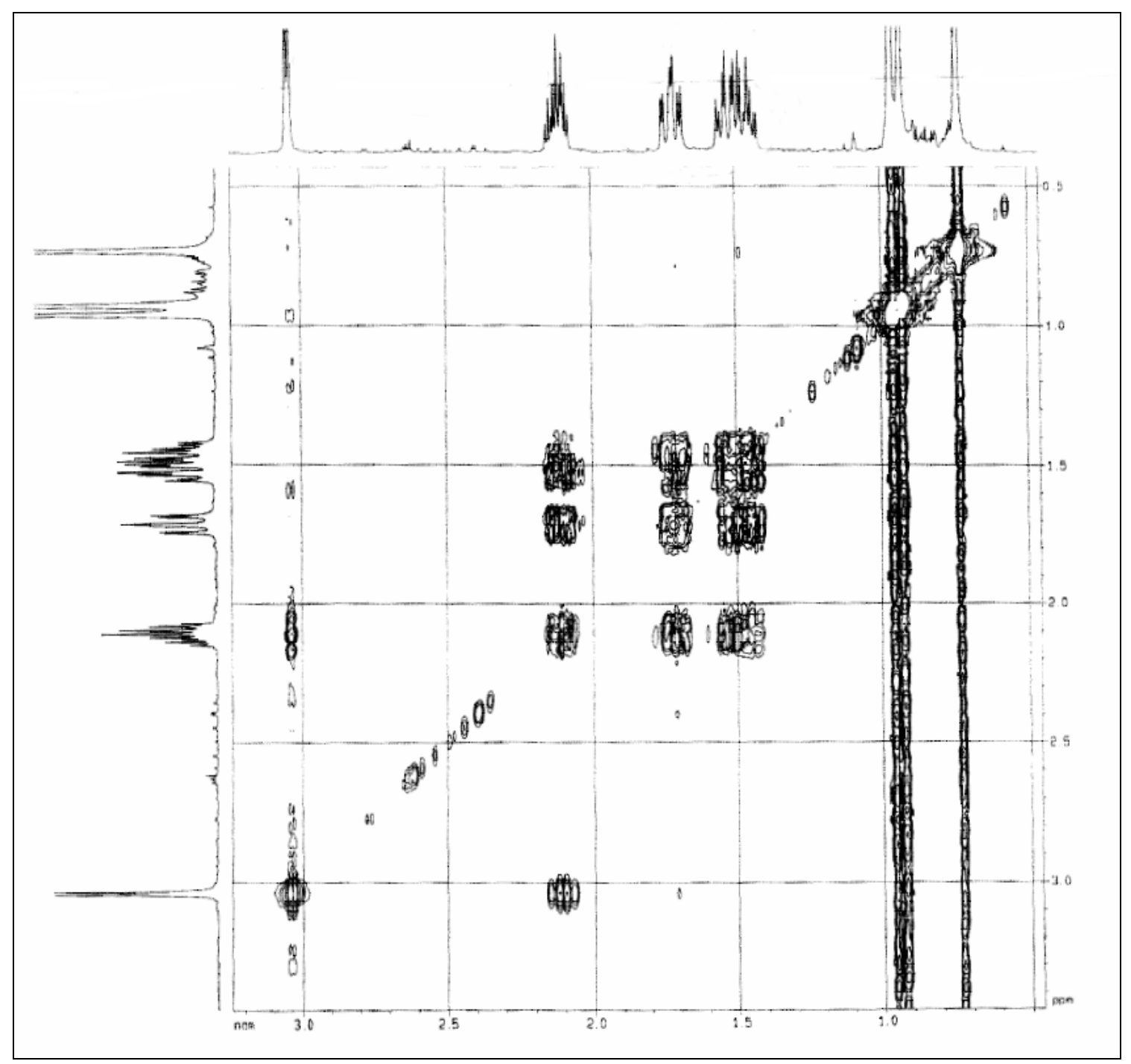


Supporting information:

Hedin-Dahlström, J.; Rosengren-Holmberg, J.P.; Legrand, S.; Wikman, S.; Nicholls, I.A. A Class II Aldolase Mimic

(1S, 4R)-(E)-3-benzylidene-1,7,7-trimethylbicyclo[2.2.1]heptan-2-one (3a)

$400 \mathrm{MHz}$ NOESY spectrum of (S)-product (3a) in $\mathrm{CDCl}_{3}$

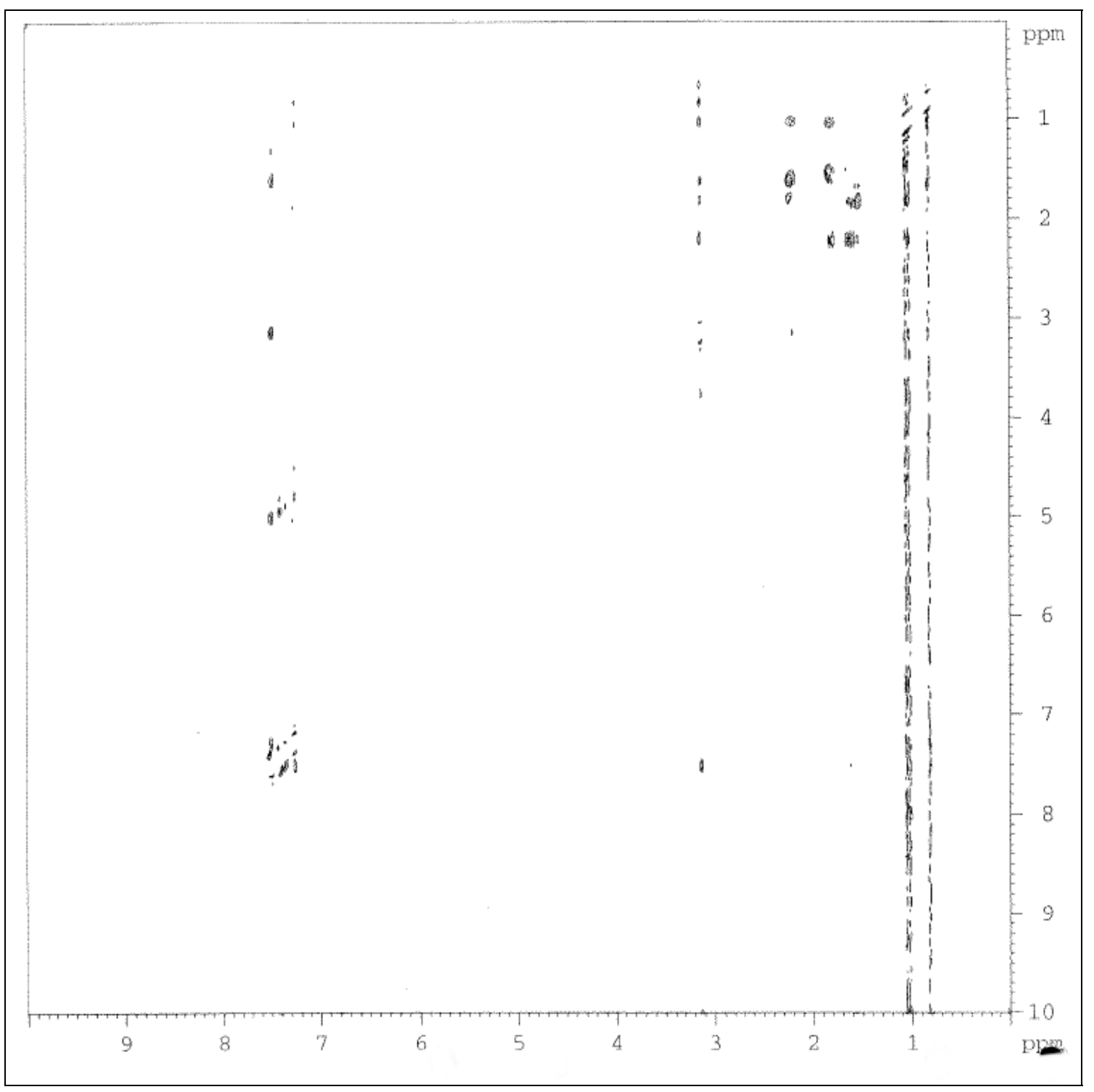


Supporting information:

Hedin-Dahlström, J.; Rosengren-Holmberg, J.P.; Legrand, S.; Wikman, S.; Nicholls, I.A. A Class II Aldolase Mimic

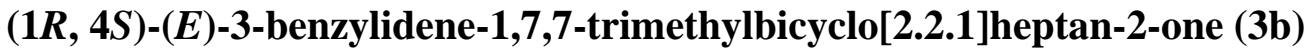

$250 \mathrm{MHz}^{1} \mathrm{H}$ NMR spectrum of $(R)$-product (3b) in $\mathrm{CDCl}_{3}$
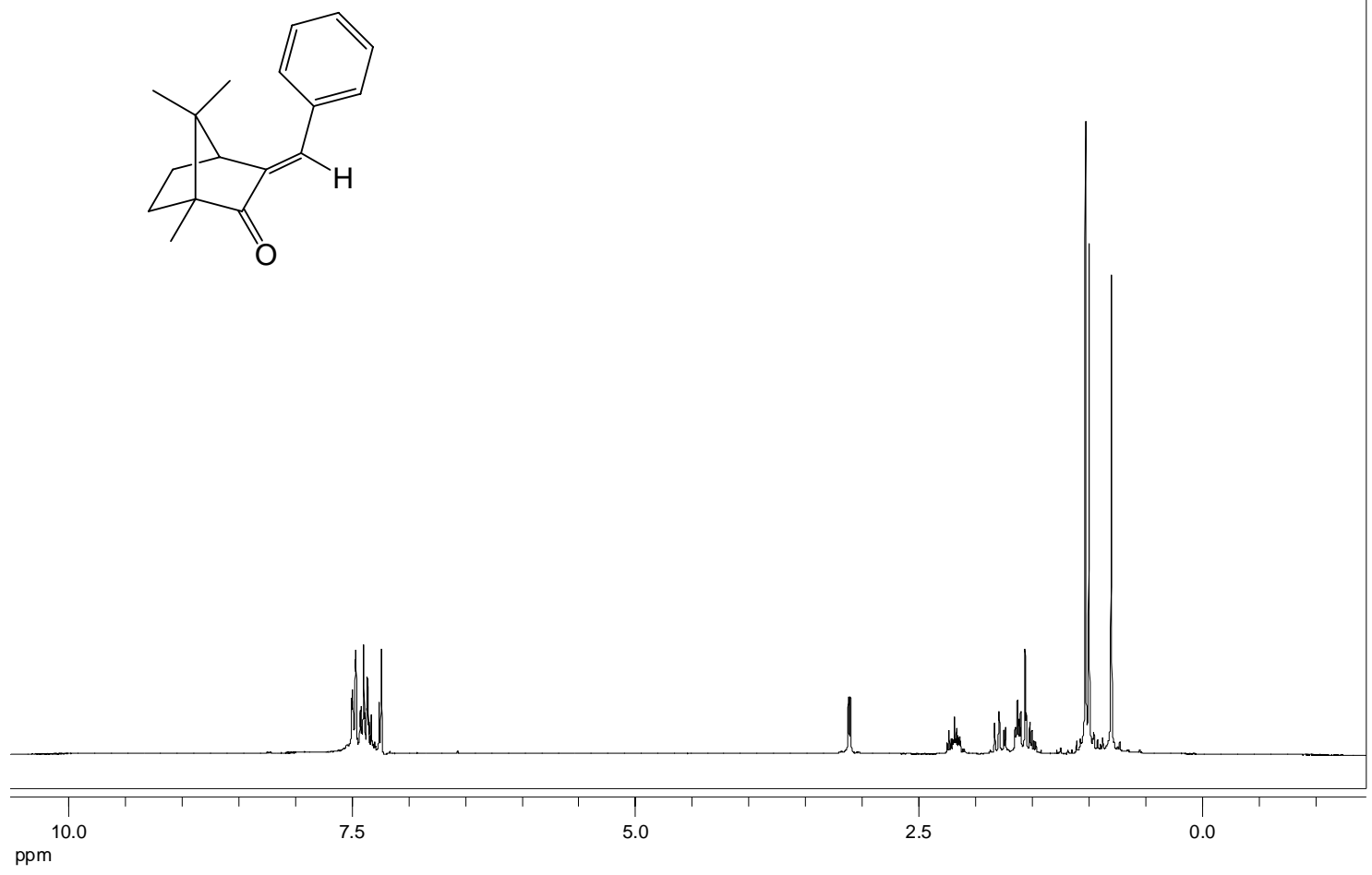

$63 \mathrm{MHz}^{13} \mathrm{C}$ NMR spectrum of $(R)$-product (3b) in $\mathrm{CDCl}_{3}$<smiles>CC12CCC(C(=Cc3ccccc3)C1=O)C2(C)C</smiles> 
Supporting information:

Hedin-Dahlström, J.; Rosengren-Holmberg, J.P.; Legrand, S.; Wikman, S.; Nicholls, I.A. A Class II Aldolase Mimic

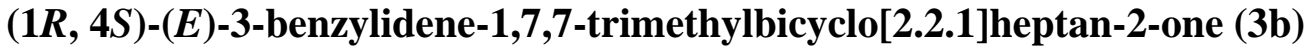

$400 \mathrm{MHz}$ COSY spectrum of $(R)$-product (3b) in $\mathrm{CDCl}_{3}$

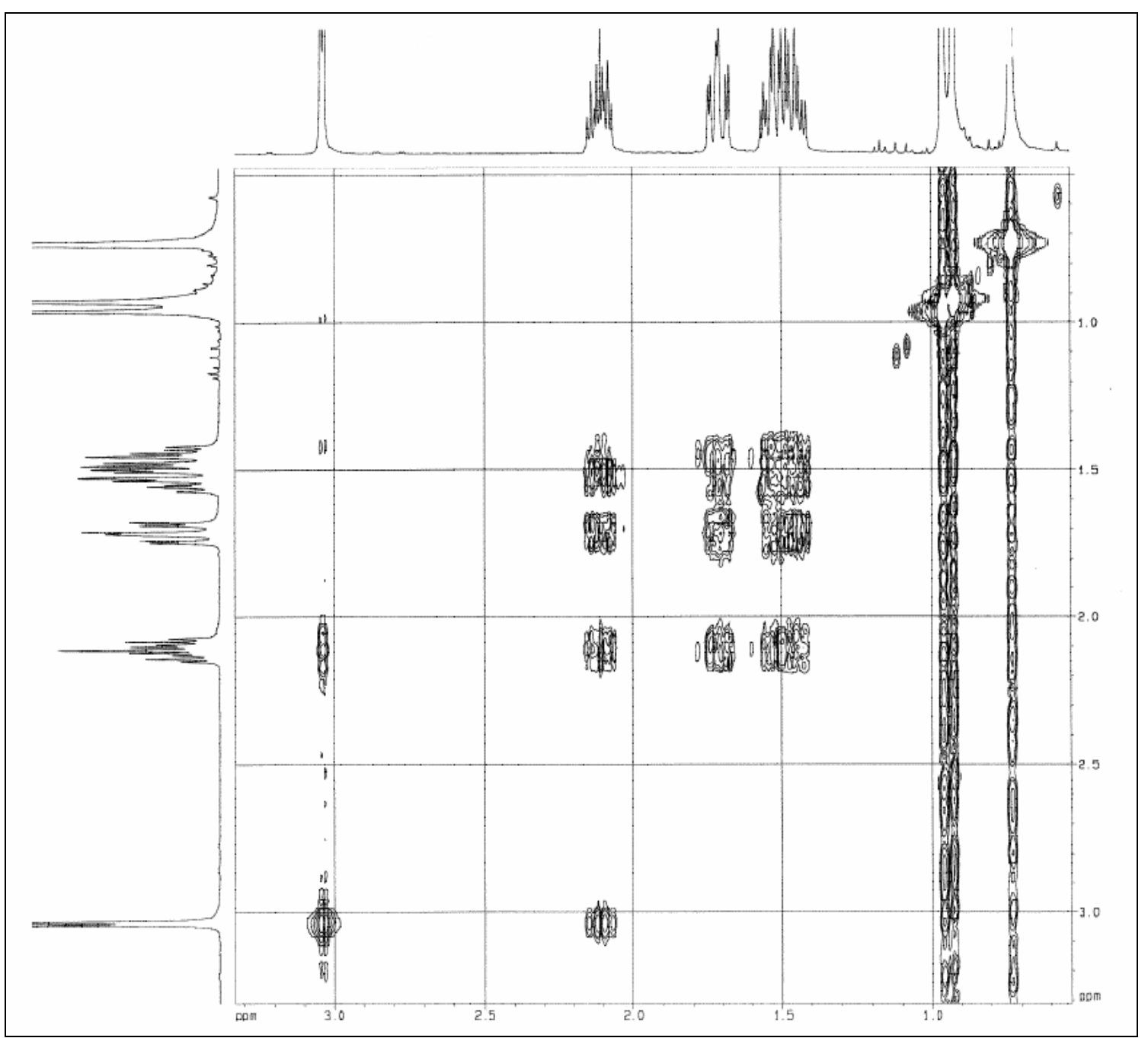


Supporting information:

Hedin-Dahlström, J.; Rosengren-Holmberg, J.P.; Legrand, S.; Wikman, S.; Nicholls, I.A. A Class II Aldolase Mimic

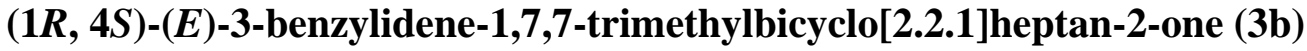

$400 \mathrm{MHz}$ NOESY spectrum of (R)-product (3b) in $\mathrm{CDCl}_{3}$

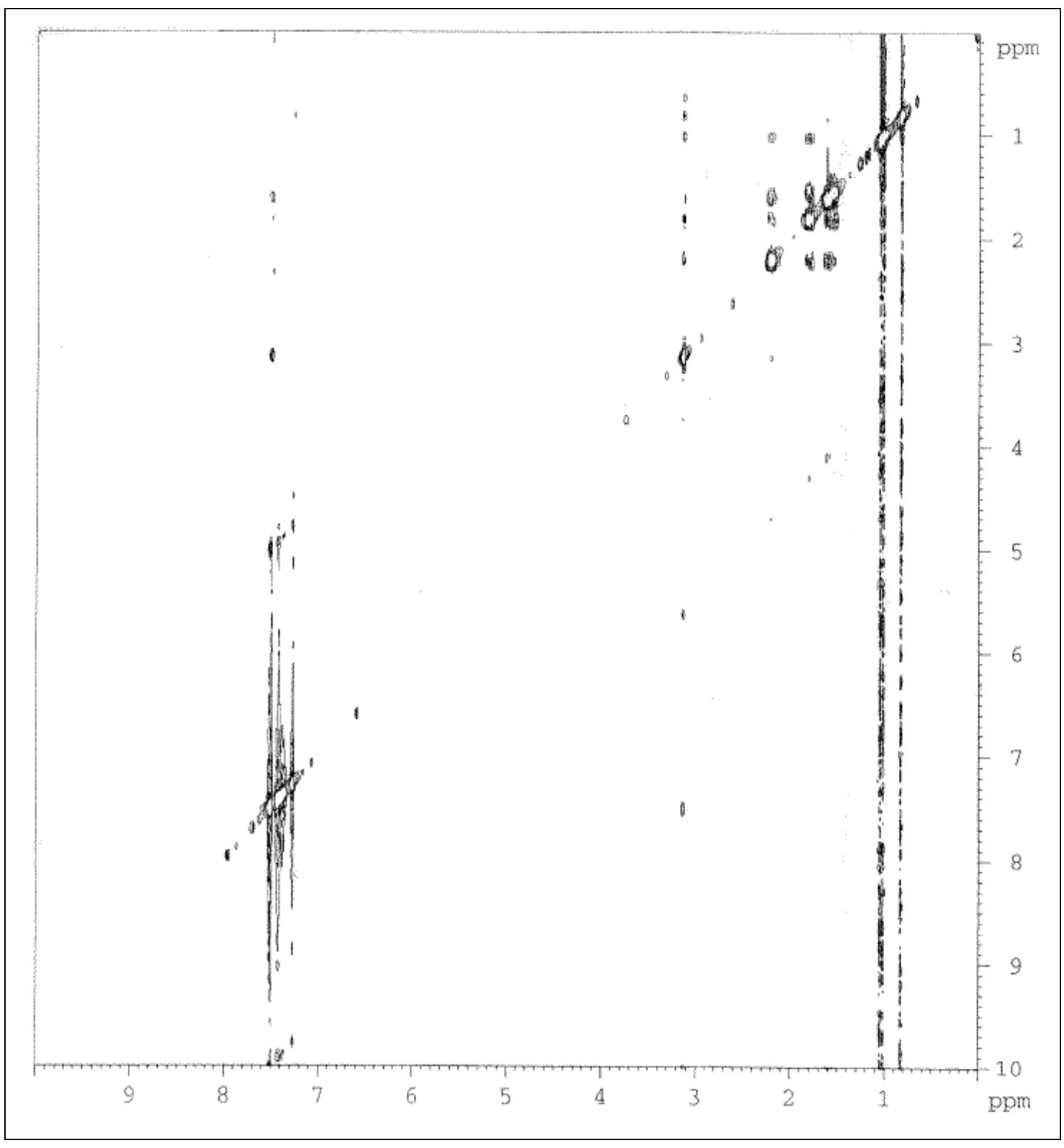


Supporting information:

Hedin-Dahlström, J.; Rosengren-Holmberg, J.P.; Legrand, S.; Wikman, S.; Nicholls, I.A. A Class II Aldolase Mimic

\section{(1S, 3S, 4S)-3-benzoyl-1,7,7-trimethylbicyclo[2.2.1]heptan-2-one (4a)}

$250 \mathrm{MHz}{ }^{1} \mathrm{H}$ NMR spectrum of (S)-TSA (4a) in $\mathrm{CDCl}_{3}$

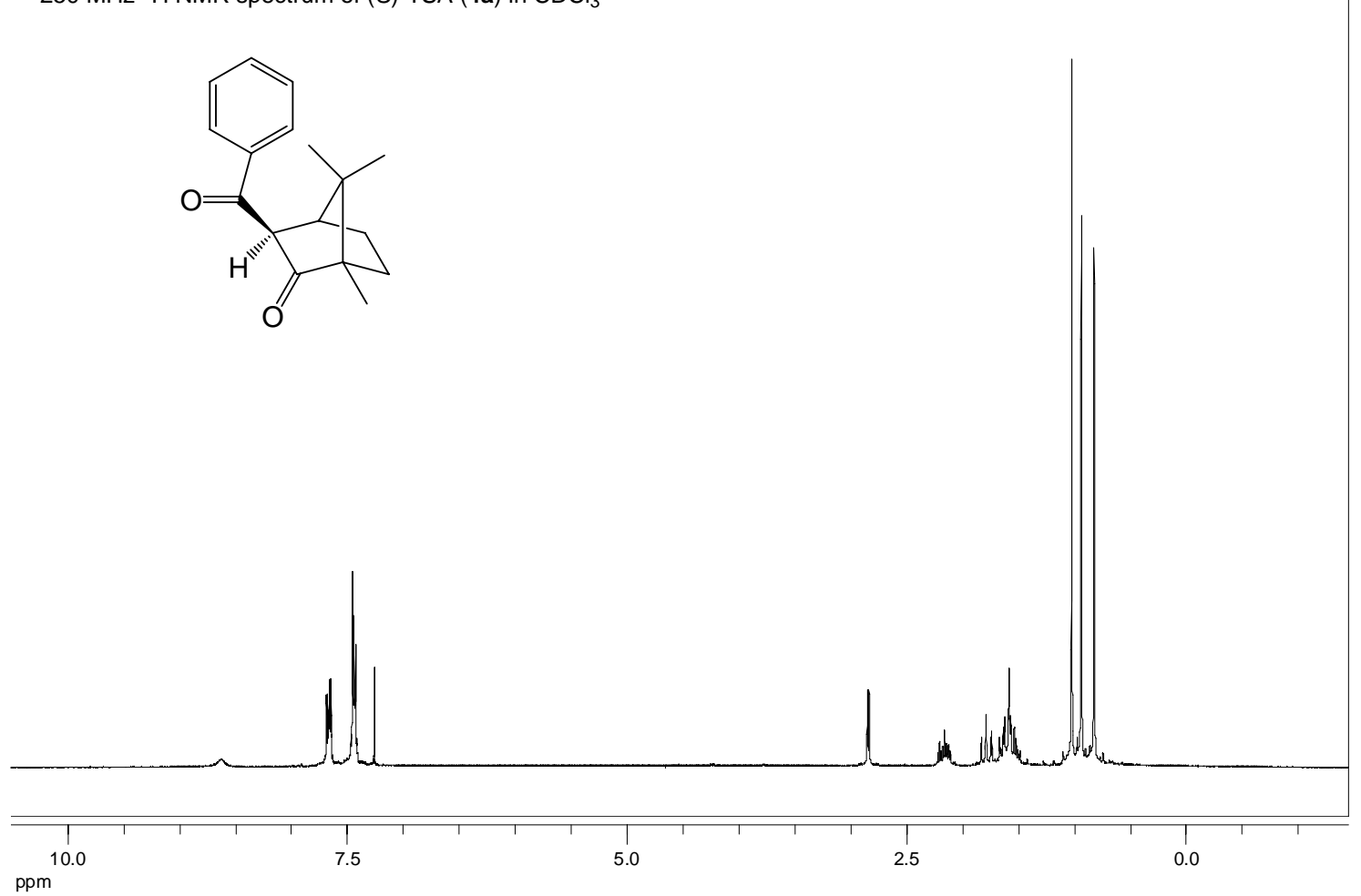

$63 \mathrm{MHz}{ }^{13} \mathrm{C}$ NMR spectrum of (S)-TSA (4a) in $\mathrm{CDCl}_{3}$
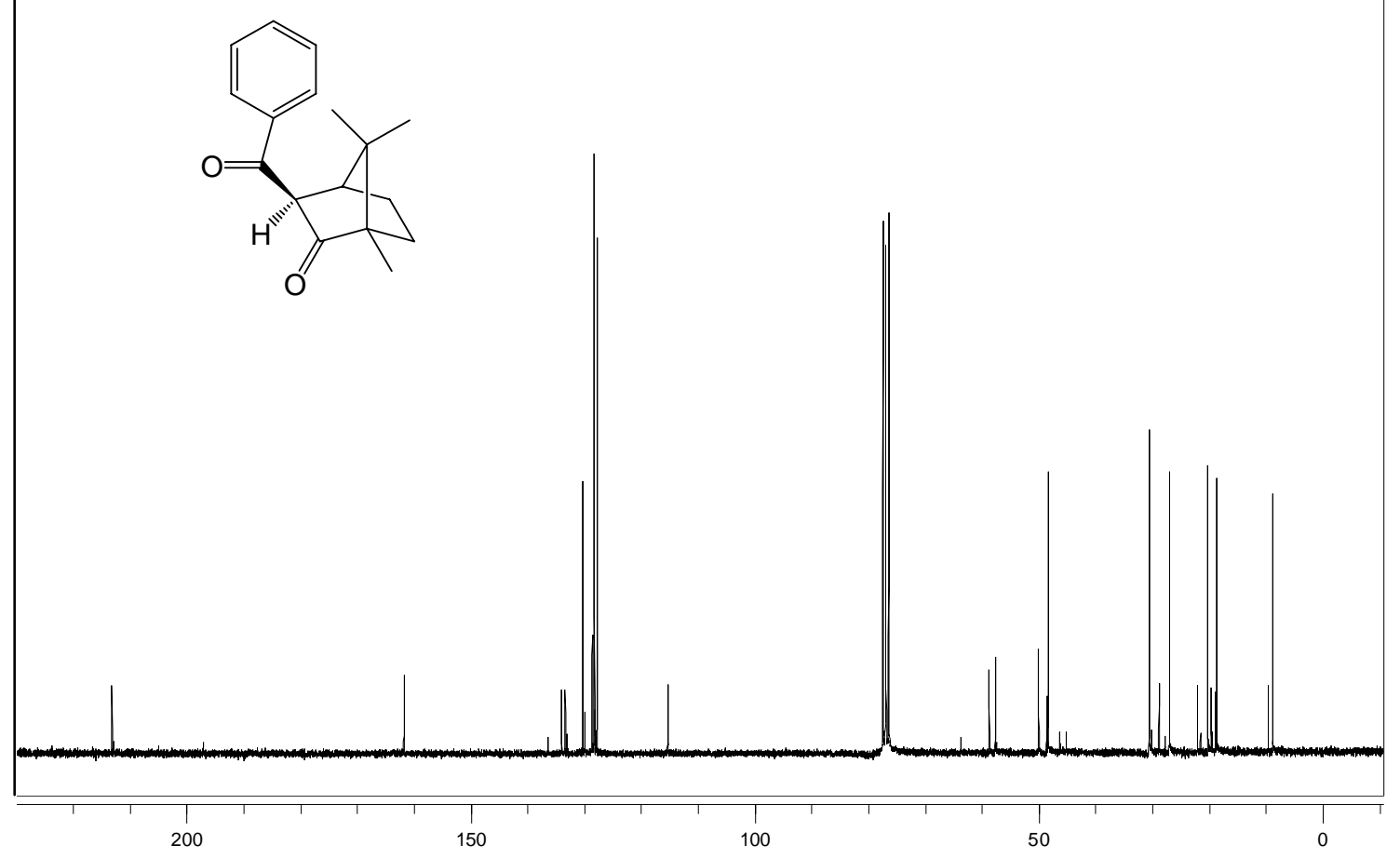
Supporting information:

Hedin-Dahlström, J.; Rosengren-Holmberg, J.P.; Legrand, S.; Wikman, S.; Nicholls, I.A. A Class II Aldolase Mimic

\section{(1S, 3S, 4S)-3-benzoyl-1,7,7-trimethylbicyclo[2.2.1]heptan-2-one (4a)}

$63 \mathrm{MHz}$ Dept-135 spectrum of (S)-TSA (4a) in $\mathrm{CDCl}_{3}$

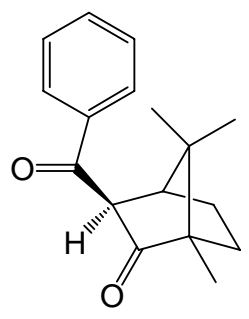

\begin{tabular}{c|c|}
\hline+1 & \\
ppm &
\end{tabular}


Supporting information:

Hedin-Dahlström, J.; Rosengren-Holmberg, J.P.; Legrand, S.; Wikman, S.; Nicholls, I.A.

A Class II Aldolase Mimic

\section{(1S, 3S, 4S)-3-benzoyl-1,7,7-trimethylbicyclo[2.2.1]heptan-2-one (4a)}

Expansion of $400 \mathrm{MHz}$ NOESY spectrum of $(4 \mathrm{a})$ in $\mathrm{CDCl}_{3}$

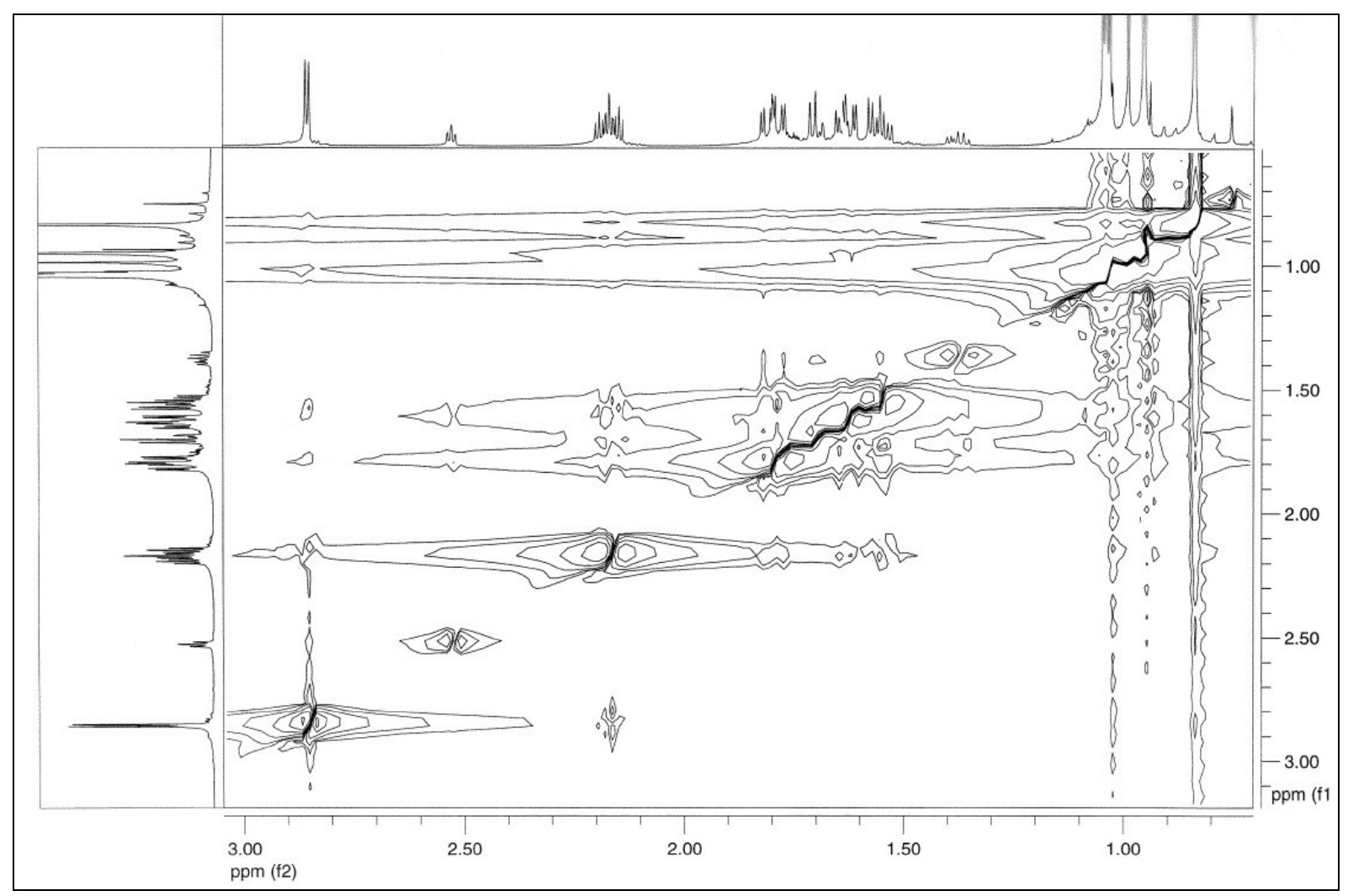


Supporting information:

Hedin-Dahlström, J.; Rosengren-Holmberg, J.P.; Legrand, S.; Wikman, S.; Nicholls, I.A. A Class II Aldolase Mimic

\section{(1R, 3R, 4R)-3-benzoyl-1,7,7-trimethylbicyclo[2.2.1]heptan-2-one (4b)}

$250 \mathrm{MHz}^{1} \mathrm{H}$ NMR spectrum of $(R)$-TSA (4b) in $\mathrm{CDCl}_{3}$
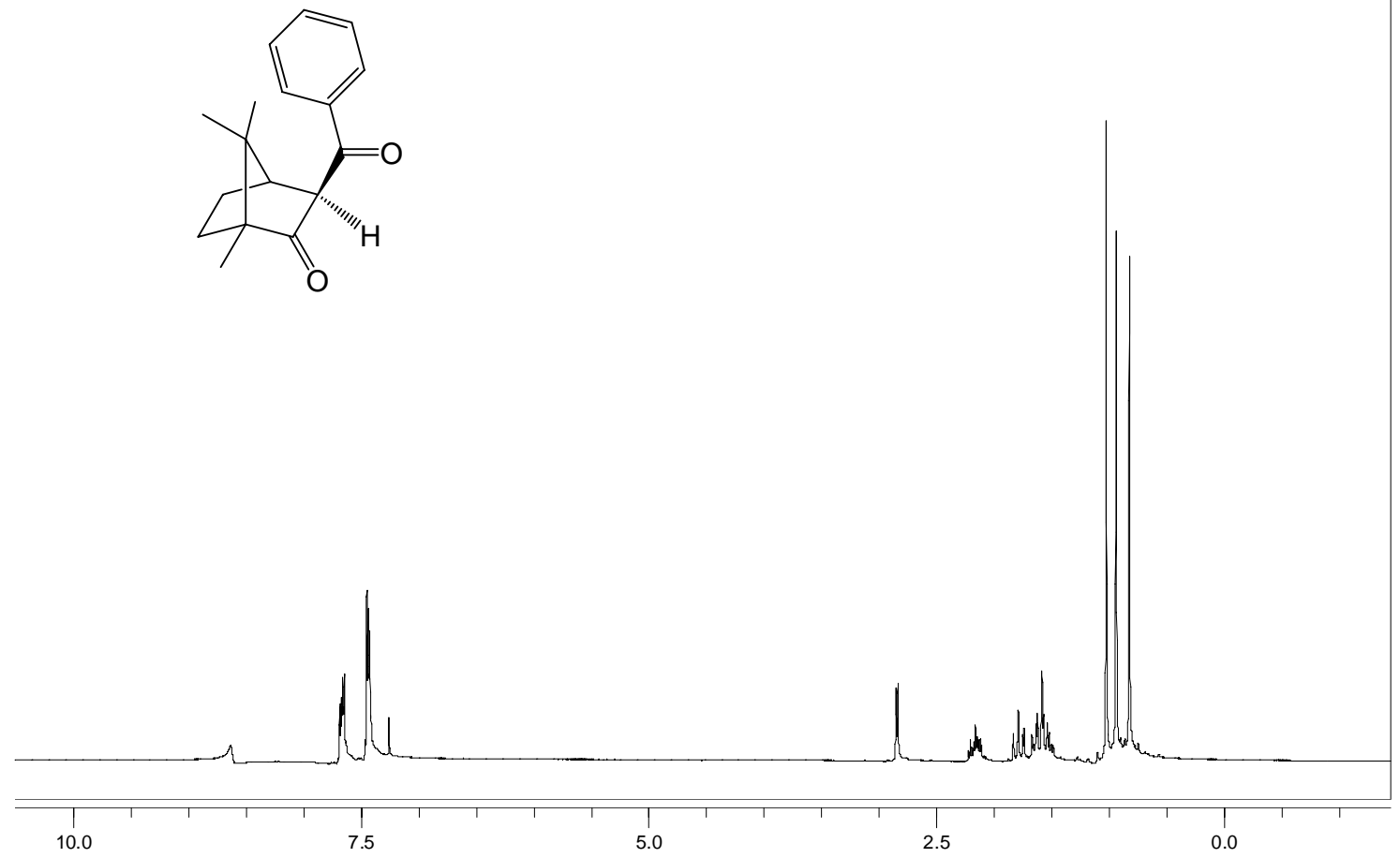

ppm

$63 \mathrm{MHz}^{13} \mathrm{C}$ NMR spectrum of (R)-TSA (4b) in $\mathrm{CDCl}_{3}$
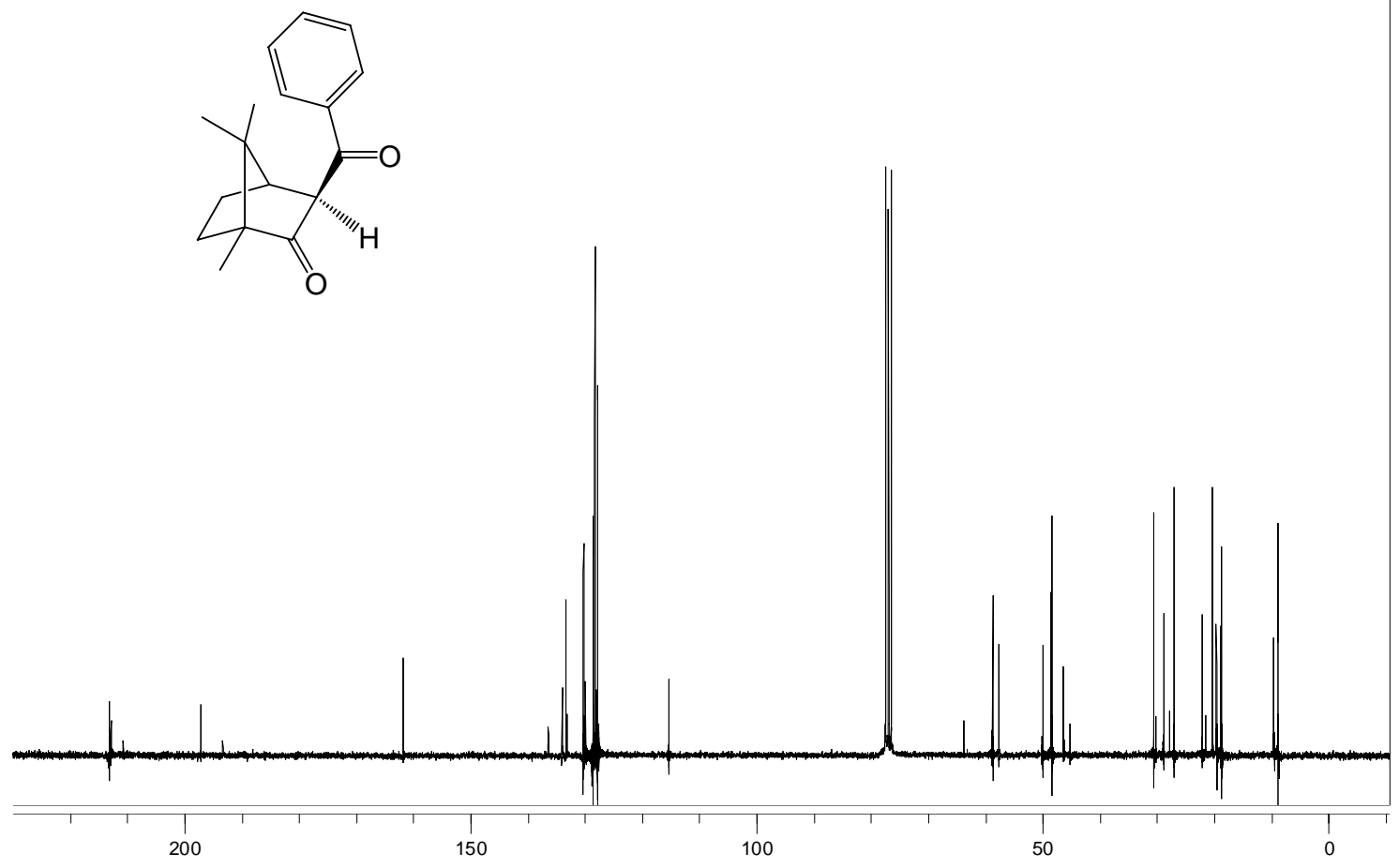
Supporting information:

Hedin-Dahlström, J.; Rosengren-Holmberg, J.P.; Legrand, S.; Wikman, S.; Nicholls, I.A.

A Class II Aldolase Mimic

\section{Proposed mechanism for keto-enol tautomerization}

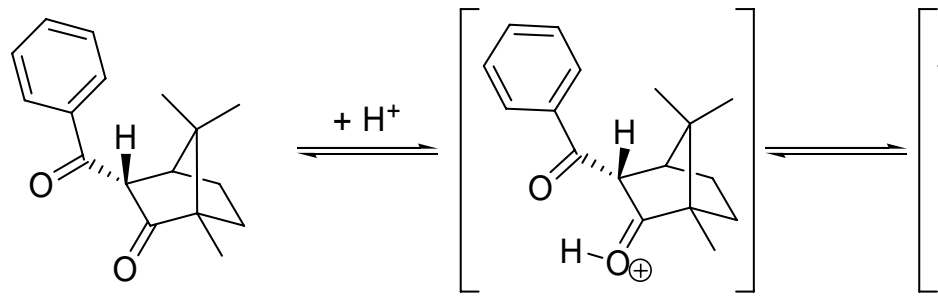

1b'

$+\mathrm{H}^{+}$

[

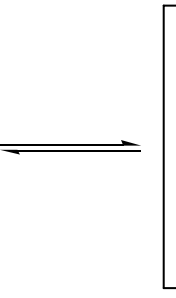

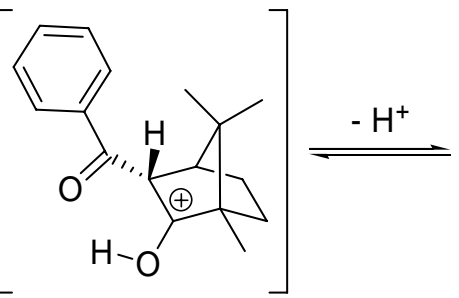

keto enol form 2

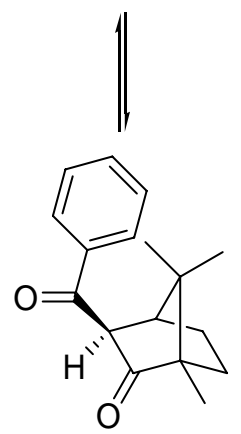

1b 
Hedin-Dahlström, J.; Rosengren-Holmberg, J.P.; Legrand, S.; Wikman, S.; Nicholls, I.A.

A Class II Aldolase Mimic

\section{VIS-titration studies}

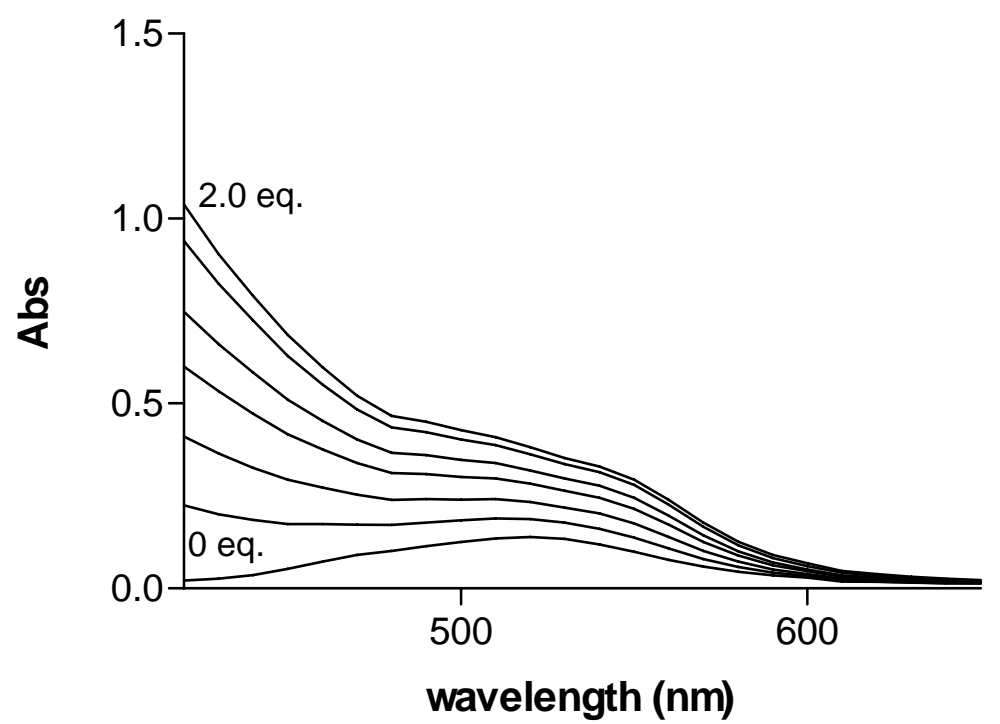

Figure 1. VIS titration of $\mathrm{Co}^{2+}$ and TSA (40 mM) in $\mathrm{MeOH}$ at r.t. Lines represent $0,0.25,0.5,0.75,1.0$, 1.5, and 2.0 eq. of TSA.

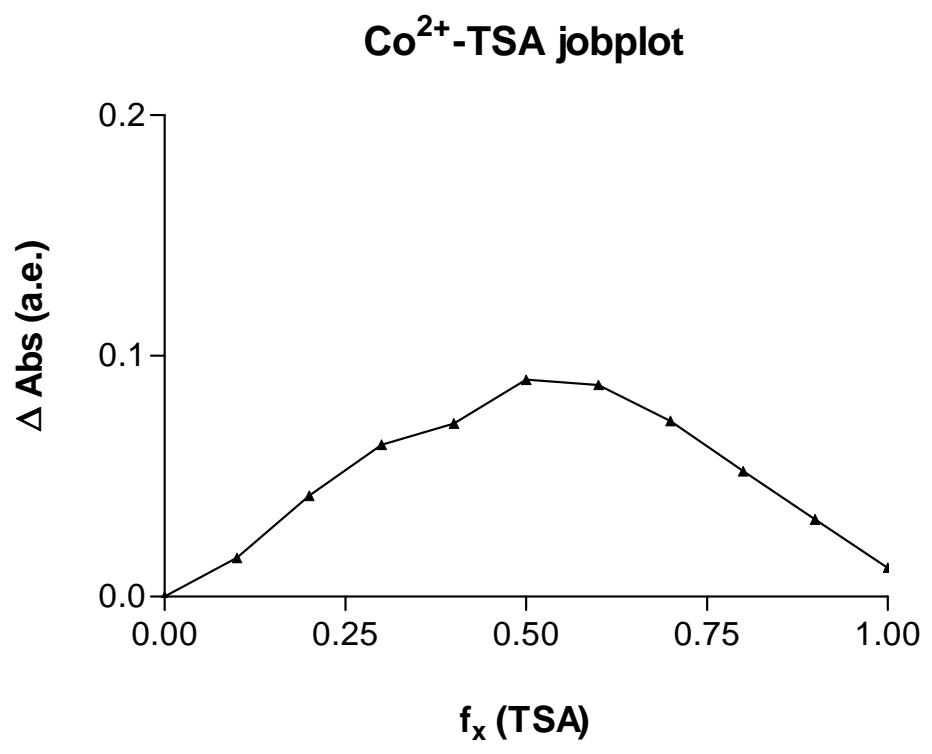

Figure: Job plot for $\mathrm{Co}^{2+}$ and TSA (4b) at $520 \mathrm{~nm}$

Total concentration: $10 \mathrm{mM}$ in $\mathrm{MeOH}$. 
Supporting information:

Hedin-Dahlström, J.; Rosengren-Holmberg, J.P.; Legrand, S.; Wikman, S.; Nicholls, I.A.

A Class II Aldolase Mimic

\section{Binding Data}

Table I: Binding of $0.015 \mathrm{mM}$ ligand:cobalt complex (1:1) in $\mathrm{MeOH}$

\begin{tabular}{lllllc}
\hline Polymer & $\boldsymbol{S}$-TSA (\%) & $\boldsymbol{R}$-TSA (\%) & $\boldsymbol{S}$-Prod (\%) & $\boldsymbol{R}$-Prod (\%) & $\mathbf{3}(\%)$ \\
\hline P0 & $12.71 \pm 0.07$ & $12.31 \pm 0.33$ & $9.59 \pm 0.10$ & $9.18 \pm 0.06$ & $0.35 \pm 0.02$ \\
P1 & $23.77 \pm 0.43$ & $23.52 \pm 0.14$ & $8.30 \pm 0.25$ & $8.16 \pm 0.06$ & $0.19 \pm 0.17$ \\
P2 & $29.35 \pm 0.74$ & $15.54 \pm 0.39$ & $10.84 \pm 0.16$ & $12.13 \pm 0.16$ & $6.26 \pm 0.48$ \\
P3 & $14.95 \pm 0.24$ & $30.25 \pm 0.24$ & $10.19 \pm 0.06$ & $12.52 \pm 0.03$ & $6.17 \pm 0.32$ \\
\hline
\end{tabular}

Table II: Binding of 0.015 mM ligand:cobalt complex (1:1) in DMF

\begin{tabular}{lccccc}
\hline Polymer & $\boldsymbol{S}$-TSA (\%) & $\boldsymbol{R}$-TSA (\%) & $\boldsymbol{S}$-Prod (\%) & $\boldsymbol{R}$-Prod (\%) & $\mathbf{3 ( \% )}$ \\
\hline P0 & $45.45 \pm 0.25$ & $47.28 \pm 0.14$ & $0.52 \pm 0.08$ & $0.19 \pm 0.02$ & $9.35 \pm 0.07$ \\
P1 & $11.21 \pm 0.24$ & $13.75 \pm 1.18$ & $0.54 \pm 0.09$ & $0.26 \pm 0.10$ & $18.49 \pm 0.16$ \\
P2 & $22.18 \pm 0.36$ & $22.65 \pm 0.13$ & $0.58 \pm 0.03$ & $0.26 \pm 0.06$ & $3.56 \pm 0.65$ \\
\hline
\end{tabular}

\section{Swelling studies}

Polymer $(1 \mathrm{~mL})$ was weighed into a $5 \mathrm{~mL}$ glass cylinder, the weight was recorded and the dry apparent density $d_{a p p}$ calculated $(\mathrm{g} / \mathrm{mL})$. DMF $(3 \mathrm{~mL})$ was added and the cylinder was sealed with a glass stopper. The increase in polymer volume was recorded after $12 \mathrm{~h}$, and the volume swelling ratio was calculated ( $S W=$ volume swollen particles/volume dry particles). The specific swelling $\left(S W_{s p}\right)$ was calculated as follows: $S W_{s p}=\frac{S W}{d_{a p p}}$ and the resulting values are reported in Table III. 
Supporting information:

Hedin-Dahlström, J.; Rosengren-Holmberg, J.P.; Legrand, S.; Wikman, S.; Nicholls, I.A. A Class II Aldolase Mimic

Table III: Specific swelling $\left(S W_{s p}\right)$ in DMF

\begin{tabular}{ll}
\hline Polymer & $\boldsymbol{S} \boldsymbol{W}_{\boldsymbol{s p}}(\mathbf{m L} / \mathbf{g})$ \\
\hline P0 & 3.36 \\
P1 & 3.62 \\
P2 & 3.71 \\
P3 & 3.70 \\
\hline
\end{tabular}

